A02-14220

\title{
AIAA 2002-1115 \\ Turbulent Primary Breakup of Round and Plane Liquid Jets in Still Air
}

K. A. Sallam, Z. Dai and G. M. Faeth

Department of Aerospace Engineering

The University of Michigan

Ann Arbor, MI 48109-2140

\section{0th Aerospace Sciences Meeting \& Exhibit 14-17 January 2002 Reno, Nevada}


AIAA 2002-1115

\title{
TURBULENT PRIMARY BREAKUP OF ROUND AND PLANE LIQUID JETS IN STILL AIR
}

\author{
K. A. Sallam, Z Z. Dai ${ }^{\dagger}$ and G. M. Faeth \\ The University of Michigan \\ Ann Arbor, MI 48109-2140, U.S.A.
}

\begin{abstract}
The formation of drops at the surface of turbulent liquids, e.g., turbulent primary breakup, was studied experimentally. Pulsed shadowgraphy and holography were used to observe the properties of the liquid surface and the drops formed by turbulent primary breakup. Measured properties included liquid surface velocities, conditions at the onset of ligament and drop formation, ligament and drop sizes, ligament and drop velocities and rates of drop formation. Phenomenological theories were used to help interpret and correlate the measurements. Present results show that the onset of ligament formation occurs once the kinetic energy of the turbulent eddies that form the ligaments exceeds the required surface tension energy of a ligament of comparable size. Subsequently, the onset of drop formation occurs once drops form at the tips of ligaments due to Rayleigh breakup. This same mechanism controls the subsequent variation of drop sizes due to turbulent primary breakup as a function of distance from the jet exit. In addition, ligament and drop velocities were associated with mean and fluctuating velocities of the liquid, and rates of drop formation could be expressed by surface efficiency factors defined as the fraction of the maximum cross stream liquid mass flux.
\end{abstract}

\section{NOMENCLATURE}

$\begin{array}{ll}\mathrm{A}_{\text {lig }} & =\text { ligament cross section area } \\ \mathrm{b} & =\text { annulus width } \\ \mathrm{C}_{\mathrm{sx}} & =\text { turbulent primary breakup coefficient } \\ \mathrm{d} & =\text { jet exit diameter } \\ \mathrm{d}_{\mathrm{h}} & =\text { jet exit hydraulic diameter } \\ \mathrm{d}_{\text {lig }} & =\text { ligament effective diameter }\end{array}$

* Graduate Student Research Assistant, Department of Aerospace Engineering.

$\dagger$ Now with G.E. Aircraft Engines, Cincinatti, OH.

$\ddagger$ A.B. Modine Professor, Department of Aerospace Engineering, Fellow AIAA, Corresponding author, Tel.: +1734-764-7202; fax: +1-734-936-0106; E-mail address: gmfaeth@umich.edu (G.M. Faeth).

Copyright (C) 2002 by G.M. Faeth. Published by the American Institute of Aeronautics and Astronautics, Inc., with permission.

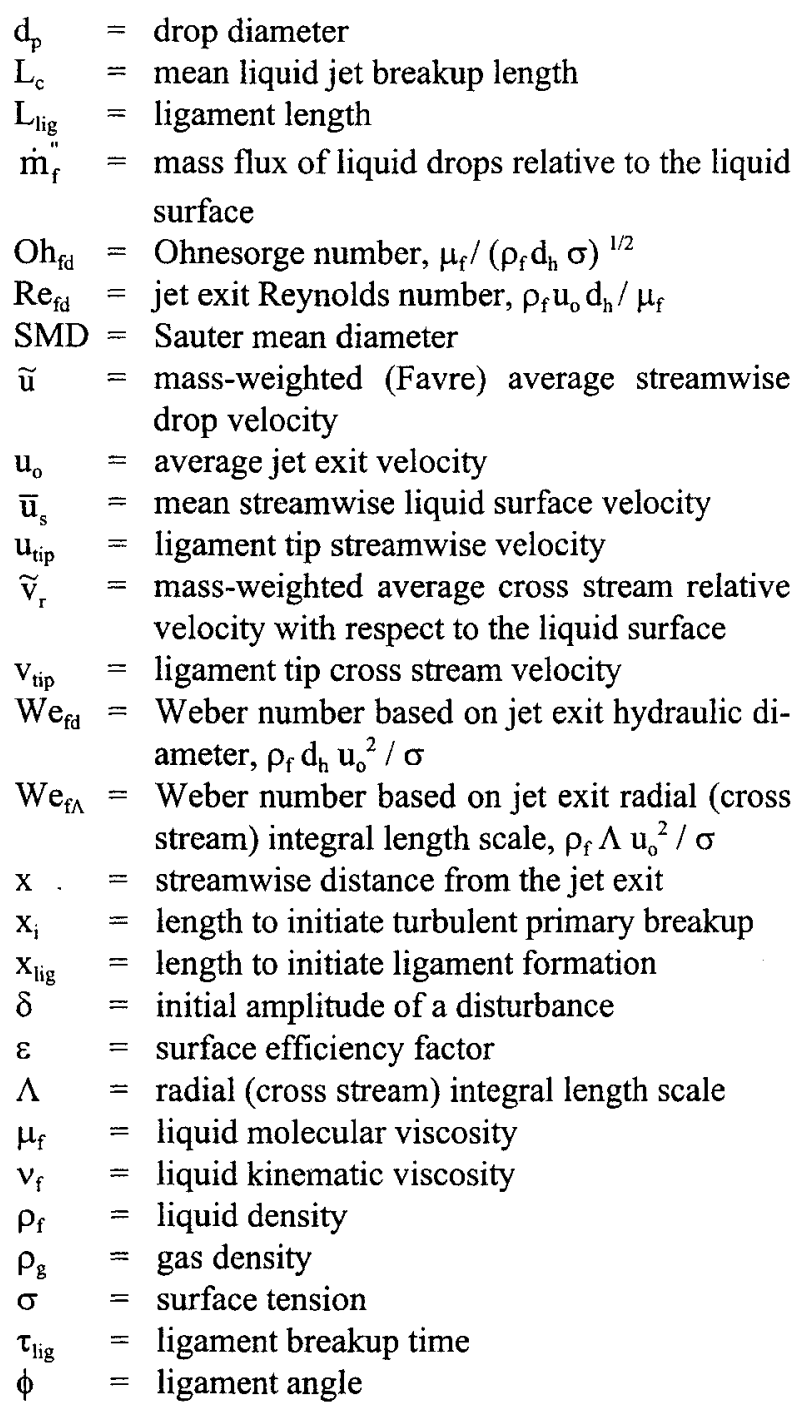

\section{INTRODUCTION}

Turbulent primary breakup is important for spray formation during a variety of industrial and natural phenomena, e.g., spray atomization and liquid jets, chutes and spillways, plunge pools, hydraulic jumps and bow sheets, breaking waves, and water falls, among others. Processes of turbulent primary breakup were identified during early flow visualization studies due to De Juhasz et al. ${ }^{1}$ and Lee 
and Spencer. ${ }^{2}$ Subsequent studies due to Schweitzer, ${ }^{3}$ Grant and Middleman, ${ }^{4}$ Phinney ${ }^{5}$ and McCarthy and Malloy ${ }^{6}$ confirmed that liquid turbulence properties affected jet stability, the onset of breakup and spray quality after breakup. Finally, Hoyt and Taylor ${ }^{7,8}$ demonstrated that aerodynamic effects were generally of secondary importance for turbulent primary breakup of turbulent liquid jets in still air at standard temperature and pressure (STP).

Earlier studies of turbulent primary breakup carried out in this laboratory include studies of liquid breakup properties at the surface of turbulent round liquid jets in still gases due to Ruff et al., ${ }^{9-11}$ Tseng et al. ${ }^{12}$ and $\mathrm{Wu}$ et al. ${ }^{13-16}$ and a study of liquid breakup properties at the surface of turbulent annular wall jets in still gases due to Dai et al. ${ }^{17,18}$ These studies resolved several aspects of turbulent primary breakup at the surface of turbulent round liquid jets in still air, as follows: aerodynamic effects were small for liquids injected into light gases (air, etc.) at STP, except far from the injector where primary and secondary breakup occur at comparable times and tend to merge, ${ }^{14}$ drop size distributions after turbulent primary breakup satisfied Simmons ${ }^{19}$ universal root normal distribution and are defined completely by the Sauter mean diameter (SMD) alone (where the SMD is the diameter of a drop having the same surface-area/volume ratio as the spray as a whole); drop velocities after turbulent primary breakup at a point are independent of drop size; the SMD of drops after turbulent primary breakup progressively increase with increasing distance from the jet exit and were effectively correlated by a phenomenological analysis of turbulent primary breakup; mean streamwise and cross stream absolute drop velocities after turbulent primary breakup were associated with mean streamwise and rms fluctuating cross stream liquid velocities at the jet exit; and conditions at the onset and end of turbulent primary breakup along the liquid surface were successfully correlated using phenomenological analysis. In addition, differences between the turbulent primary breakup properties of round free jets and annular wall jets were observed, which raises questions about the role of the geometry change in causing these differences that have not yet been resolved.

Unfortunately, some important features of turbulent primary breakup of round turbulent liquid jets in still gases were not resolved during this work. First of all, the rates of drop formation due to turbulent primary breakup along the liquid surface were not found. In addition, a closely related property of drop formation rates, the cross stream drop velocity relative to the liquid surface, was not found. Finally, drop velocity information was mainly gathered for the region near the jet exit leaving substantial uncer- tainties about drop velocities in the critical region near the end of the turbulent liquid column.

In view of current status of the understanding of turbulent primary breakup for round and annular free jets, the objectives of the present study were to complete new measurements of the spatial and dynamic properties of ligaments along the surface of the annular and round turbulent free jets and investigate earlier theories ${ }^{13}$ concerning the role of the classical Rayleigh breakup mechanism in the formation of drops from the ligaments and to complete new measurements of the rates of turbulent primary breakup along the surface of turbulent annular and round liquid jets in still gases, seeking also to resolve the associated streamwise and cross stream drop velocities relative to the liquid surface and including conditions near the end of the liquid column.

\section{EXPERIMENTAL METHODS}

\section{Apparatus}

Two test apparatus were employed during the present study: (1) an annular jet apparatus and (2) a round jet apparatus. Figure 1 is a sketch of the annular jet apparatus. Annular liquid jets having aspect ratios larger than 20:1 were used in order to avoid the problems of defining end effects of finite-width plane jets used to approximate plane liquid jets. Pressure injection was used to feed the test liquids from a cylindrical storage chamber through an annular nozzle directed vertically downward into still air at atmospheric pressure. The storage chamber had an inside diameter and length of 190 and $305 \mathrm{~mm}$, respectively. The annular nozzle had a $50 \mathrm{~mm}$ inner diameter with annulus widths of 3.55 and $6.75 \mathrm{~mm}$ to yield annular liquid jets, having aspect ratios greater than 23 to approximate plane free jets. The nozzle passages had rounded entries (radii of curvature of 1.5 times the annulus width) followed by annular passages having length-todiameter ratios greater than 40:1 to both avoid developing cavitating flows and to insure fully-developed turbulent pipe flow at the jet exit for sufficiently large Reynolds numbers, as discussed by Wu et al. ${ }^{15}$ and references cited therein. The core of the annulus was well ventilated to prevent collapse of the annulus sheet after leaving the injector. The round jet apparatus was similar to the annular jet apparatus. The nozzle had a smooth rounded entrance (radius of curvature equal to the nozzle passage radius) followed by round constant area passages having length-to-diameter ratios greater than $40: 1$ to help insure fully-developed turbulent pipe flow at the jet exit. ${ }^{15}$

The high-pressure air was stored in an accumulator having a volume of $0.12 \mathrm{~m}^{3}$ on the upstream side of the sole- 
noid valve, with provision for accumulator air pressures up to $1900 \mathrm{kPa}$. A baffle at the air inlet prevented undesirable mixing between the air and the test liquid. In order to accommodate rigidly-mounted optical instrumentation. the nozzle assembly could be traversed up to $1.5 \mathrm{~m}$ in the vertical direction in order to measure the flow structure. Liquid injection times of $100-400 \mathrm{~ms}$ were long compared to flow development times of 6-70 ms. Present optical measurements required less than $0.1 \mathrm{~ms}$ for triggering and data acquisition which did not impose any limitation on flow times. Liquid jet velocities were calibrated as a function of nozzle pressure drop by measuring liquid surface velocities using double pulse shadowgraphs.

\section{Instrumentation}

Instrumentation consisted of single- and double-pulse shadowgraphy and off-axis holography. Shadowgraphy provided flow visualization, liquid surface velocities, the locations of the onset of ligament and drop formation, drop sizes and drop velocities along the liquid surface.

The light sources for these systems were two frequencydoubled YAG lasers (Spectra Physics model GCR-130, $532 \mathrm{~nm}$ wavelength, $7 \mathrm{~ns}$ pulse duration, and up to 300 $\mathrm{mJ}$ per pulse) that could be controlled to provide pulse separations as small as $100 \mathrm{~ns}$. These measurements were obtained with an open camera shutter under darkroom conditions so that the laser pulse duration controlled the exposure time and was short enough to stop liquid surface and drop motion. The use of different pulse strengths for double-pulse shadowgraphs allowed directional ambiguity to be resolved. Polaroid types 55 and 57 black and white films were used to record the images. Data was obtained from the shadowgraphs by mounting them on a computer controlled $x-y$ traversing system (having a 1 $\mu \mathrm{m}$ resolution) and observing the images using a SONY $\mathrm{CCD}$ video camera (model $\mathrm{XC}-77$ ). The overall arrangement allowed drops as small as $5 \mu \mathrm{m}$ diameter to be observed and as small as $10 \mu \mathrm{m}$ diameter to be measured with $10 \%$ accuracy.

Measurements of mean drop velocities were based on the motion of the centroid of the drops; finding these averages was simplified because drop velocity distributions were essentially uniform. The experimental uncertainties ( $95 \%$ confidence) were smaller than 5 and $20 \%$, for the mean streamwise and cross stream drop velocities, respectively. Mass-averaged cross stream drop velocities relative to the liquid surface were found by summing the relative cross stream velocities of 40-400 drops with experimental uncertainties ( $95 \%$ confidence) smaller than $20 \%$. In all cases, experimental uncertainties were dominated by sampling limitations.
Off-axis single-pulse holography was used to find drop liquid fluxes at the liquid surface (the rate of drop formation) due to turbulent primary breakup, eliminating the depth of field correction problem of conventional photographic particle sizing techniques. The arrangement is based on the Spectron Development Laboratories Model HTRC-5000 holographic camera and allowed drops as small as $5 \mu \mathrm{m}$ diameter to be seen and drops as small as $10 \mu \mathrm{m}$ diameter to be measured with $10 \%$ accuracy. The holograms were obtained in a darkened room using AGFA 8E75HD-NAH holographic plates with a $100 \mathrm{~mm}$ $\mathrm{x} 125 \mathrm{~mm}$ film format. The holograms were reconstructed using a $35 \mathrm{~mW}$ HeNe laser, with the laser beam collimated and passed through the hologram to provide a real image of the spray.

The rate of drop formation along the surface was obtained by measuring the volume of the drop liquid within an annular segment defined by the angle between two alignment pins (positioned outside the spray-containing region) and the axis of the liquid jet, with the distance above the mean position of the liquid surface given by the distance the drops move at the mean relative velocity with respect to the liquid surface for a preselected sampling time. Experimental uncertainties of liquid flux measurements due to the definition of irregular objects as ellipsoids are difficult to quantify; otherwise, 40-60 sample volumes were evaluated to yield mean radial liquid drop fluxes with experimental uncertainties ( $95 \%$ confidence) less than $30 \%$, mainly dominated by sampling limitations.

\section{Test Conditions}

The experiments involved measurements of both annular and round fully-developed turbulent jets. Present tests were limited to water and ethanol injected into still air, however, past work ${ }^{13-18}$ concerning round free jets and plane wall jets in still air has shown that effects of variations of liquid and gas properties can be treated effectively by the dimensionless parameters used to summarize the present test results. Present test conditions for round and annular free jets are summarized in Table 1 and 2, respectively. The Reynolds number range implies that effects of turbulent primary breakup dominated the present results. The small jet exit Ohnesorge numbers imply that direct effects of liquid viscosity on liquid breakup were small.

\section{RESULTS AND DISCUSSION}

\section{Ligament characteristics}

A typical ligament at the surface of an annular free jet is shown in Fig. 2. Notably, the geometrical appearance of 
ligaments does not lend itself easily to a simple prescription for ligament shapes. As a result, an idealization of the geometry of the ligaments was necessary. This was done using an approach similar to Merrill and Sarpkaya. ${ }^{20}$ The definitions of ligament length, effective diameter, and ligament angle, are illustrated in Fig.2. The ligament effective diameter is calculated from the measured ligament length and the ligament projected area on the shadowgraph as:

$$
\mathrm{d}_{\text {lig }}=\mathrm{A}_{\text {lig }} / \mathrm{L}_{\text {lig }}
$$

Measurements of 250 ligaments along the surface of round and annular free jets yielded the probability density distribution of ligament angle as shown in Fig. 3. The probability density distribution of ligament angle appears to be roughly Gaussian, with a mean value of $105 \mathrm{deg}$ and a standard deviation of $32 \mathrm{deg}$. The probability density distribution function of the ligament angle indicates that the ligaments are nearly randomly oriented which suggests small aerodynamic effects, in agreement with past assessments of aerodynamic effects on turbulent primary breakup for similar conditions. ${ }^{14}$

\section{Ligament breakup mechanism}

Two mechanisms for ligament breakup were observed: (1) ligament tip (Rayleigh) breakup and (2) ligament base (turbulent) breakup. Typical shadowgraphs of ligament tip (Rayleigh) breakup are illustrated in Fig.4. Corresponding typical shadowgraphs of ligament base (turbulent) breakup are illustrated in Fig. 5. The first mechanism is dominant and is observed roughly $90 \%$ of the time. In this case, breakup of the ligament tip occurred similar to classical Rayleigh breakup, where the ligaments act like liquid jets emerging from the liquid surface. The resulting drops tend to have drop/ligament diameter ratios close to 1.9 which was proposed by Tyler ${ }^{21}$ as roughly the diameter ratio for Rayleigh breakup. The second mechanism of drop formation, which was occasionally observed (less than $10 \%$ of the time), involved breakup of the ligament at its base due to the turbulent cross stream velocity fluctuations which change the flow direction at the base of the ligament before Rayleigh breakup can occur at the ligament tip. This mechanism is characterized by the formation of large, irregular, ligament-like drops and yields larger drop/ligament diameter ratios, $\mathrm{d}_{\mathrm{p}} / \mathrm{d}_{\mathrm{lig}}$ than ligaments associated with Rayleigh breakup.

The probability distribution of drop/ligament diameter ratio, $\mathrm{d}_{\mathrm{p}} / \mathrm{d}_{\mathrm{lig}}$, had a mean value of 2.1 and a standard deviation of 0.78 . This shows that the ligament tip breakup mechanism is the dominant mechanism for drop forma- tion because the majority of the drop/ligament diameter ratio measurements yielded values near 1.9, typical of Rayleigh breakup, rather than the larger values $(>3)$ typical of large ligament-like drops formed through the ligament base breakup mechanism.

A second test for the Rayleigh breakup hypothesis involved comparison of the ligament breakup time to the Rayleigh breakup time. This test was performed at the onset of breakup conditions. The length to initiate the onset of ligaments and the onset of drops (i.e. onset of breakup) were measured for annular and round free jets as shown on Fig. 6. The times required for ligament breakup were then calculated as follows:

$$
\tau_{\text {lig }}=\left(x_{i}-x_{\text {lig }}\right) / u_{o}
$$

The ligament breakup time normalized by the Rayleigh breakup time, $\left(\rho_{\mathrm{f}} \mathrm{d}_{\mathrm{lig}}{ }^{3} / \sigma\right)^{1 / 2}$, taken from Weber ${ }^{22}$ also is plotted on Fig. 6 as a function of the Weber number for annular and round free jets. Clearly, the ratio of the ligament breakup time to the Rayleigh breakup time is on order of unity in Fig. 6, which further supports Rayleigh drop formation mechanism from ligaments.

Finally, measurements of ligament slenderness ratio, i.e., ligament length/diameter ratio at the instant of breakup, provide another important test of the Rayleigh breakup mechanism of drop formation from ligaments. The theoretical prediction of the length of a eylindrical jet undergoing Rayleigh breakup is given by ${ }^{22}$ :

$$
\mathrm{L}_{\mathrm{c}} / \mathrm{d}=[\ln (\mathrm{d} / 2 \delta)]\left(3 \mathrm{We}_{\mathrm{fd}} / \mathrm{Re}_{\mathrm{fd}}+\mathrm{We}_{\mathrm{fd}}^{1 / 2}\right)
$$

where $\delta$ is the initial amplitude of a disturbance and the parameter $[\ln (d / 2 \delta)]$ must be determined experimentally. Weber ${ }^{22}$ reported a value of 12 for this parameter. The Weber/Reynolds ratio was small compared to the square root of the Weber number for the present test conditions. Consequently, the ligament slenderness ratio $\mathrm{L}_{\text {lig }} / \mathrm{d}_{\mathrm{lig}}$ at the time of breakup is plotted in Fig. 7 as a function of the square root of the Weber number (based on ligament diameter and ligament tip speed) as suggested by Eq. 3. The best fit correlation of the data is given by:

$$
\mathrm{L}_{\text {lig }} / \mathrm{d}_{\text {lig }}=0.59\left(\rho\left(\mathrm{u}_{\mathrm{tip}}{ }^{2}+\mathrm{v}_{\text {tip }}{ }^{2}\right) \mathrm{d}_{\text {lig }} / \sigma\right)^{0.32}
$$

The standard deviation of the power of Eq. 4 is $28 \%$ and the correlation coefficient of the fit is 0.82 . The power of the Weber number is not 0.5 as suggested by Eq. 3 but the difference is not large in view of the relatively large uncertainties of the measurements of the ligament 
breakup properties using double-pulse shadowgraphy. In addition, Eq. 4 suggests a value of 0.59 for the parameter $[\ln (d / 2 \delta)]$ in contrast to a value of 12 reported earlier by Weber ${ }^{22}$ as noted earlier. This result indicates that $\mathrm{d}_{\mathrm{lig}} / \delta=$ 3.6 for ligament breakup. This implies a disturbance of order of magnitude of the ligament diameter itself which supports the earlier ideas of Wu and Faeth ${ }^{16}$ that turbulent primary breakup is caused by turbulent eddies escaping from the surface of the liquid jet.

\section{Ligament and drop sizes along the surface}

An expression for the variation of the ligament diameter resulting from turbulent primary breakup as a function of the distance from the jet exit for annular and round free jets can be developed following methods used earlier for the variation of the SMD of drops formed by turbulent primary breakup for both round free jets ${ }^{13}$ and annular wall jets. ${ }^{17}$ It was assumed that the ligament diameter was dominated by the largest ligament that can be formed at a given position, that effects of liquid viscosity are small, and that the ligament diameter is proportional to the characteristic eddy size. Then the following expression for the variation of $\mathrm{d}_{\mathrm{lig}} / \Lambda$ with distance from the jet exit is obtained:

$$
\mathrm{d}_{\mathrm{igg}} / \Lambda=\mathrm{C}_{\mathrm{sx}}\left(\mathrm{x} /\left(\Lambda \mathrm{We}_{\mathrm{f} \Lambda}^{1 / 2}\right)\right)^{2 / 3}
$$

where $\mathrm{C}_{\mathrm{sx}}$ is an empirical parameter on the order of unity. Data for ligament size at the surface of both the round and annular free jets are plotted in Fig. 8 as suggested by Eq. 5. The best fit correlation for ligament size is given by:

$$
\mathrm{d}_{\operatorname{lig}} / \Lambda=0.38\left(\mathrm{x} /\left(\Lambda \mathrm{We}_{\mathrm{f} \Lambda}^{1 / 2}\right)\right)^{0.61}
$$

The standard deviations of the coefficient and power of Eq. 6 are 8 and $9 \%$, respectively, and the correlation coefficient of the fit is 0.95 . The power of the normalized streamwise variable in Eq. 6 is nearly the same as the theoretical value given by Eq. 5 (i.e. 0.66 ) whereas the coefficient is of order of magnitude unity as expected. The reasonable value of the empirical coefficient and power of Eq. 6 and the large correlation coefficients of the fit, help to support the physical ideas used to develop these expressions.

Drop sizes resulting from Rayleigh breakup of ligaments are roughly twice the size of the ligaments as expected. Assuming that the SMD is proportional to the largest drops formed in this way, yields a correlation of SMD/ $\Lambda$ similar to the results reported by $\mathrm{Wu}$ and Faeth. ${ }^{14}$

\section{Drop velocities}

The values of both streamwise and cross stream drop velocities are observed to increase in magnitude with increasing $\mathrm{d}_{\mathrm{p}} / \mathrm{SMD}$ initially, but then remain nearly unity (within experimental uncertainties) for $0.3<\mathrm{d}_{\mathrm{p}} / \mathrm{SMD}$. Consideration of the behavior of the small drops, however, suggests that their velocities tend to be smaller than the rest due to their rapid relaxation toward the local gas velocity. In addition, the effect of small drops on the momentum exchange between the phases is not very important because they have correspondingly small inertias. Thus, assuming uniform drop velocity distributions after turbulent primary breakup at each point along the surface appears to be justified based on the present measurements. Similar conclusions were obtained from analysis of earlier drop velocity measurements after turbulent primary breakup for annular wall jets due to Dai et al. ${ }^{17,18}$

Mean streamwise and mean relative cross stream drop velocities are a function of normalized distance from the jet exit. The results plotted in Fig. 9 show first of all that the normalized measurements for round and plane turbulent liquid jets are essentially the same. Next, mean streamwise drop velocities are closely associated with the local streamwise liquid surface velocity, with all the measurements illustrated in Fig. 9 yielding

$$
\widetilde{\mathrm{u}} / \overline{\mathrm{u}}_{\mathrm{s}}=0.89
$$

with a standard deviation of this ratio of 0.03 , over the length of the liquid column. Also $\bar{u}_{s} / u_{o}$, is nearly independent of streamwise distance over the length of the liquid column for a round jet or the length of the liquid sheet for an annular jet and is equal to 0.89 . The results for annular wall jets from Dai et al. ${ }^{17}$ are shown along with the results for annular and round free jets. The relative cross stream velocities for all three flows exhibit essentially the same streamwise variation. The correlation of the relative cross stream velocities, $\widetilde{v}_{\mathrm{r}} / \overline{\mathrm{u}}_{\mathrm{s}}$, is described by the following:

$$
\widetilde{\mathrm{v}}_{\mathrm{r}} / \overline{\mathrm{u}}_{\mathrm{s}}=0.055, \quad \mathrm{x} /\left(\Lambda \mathrm{We}_{\mathrm{f} \Lambda}^{0.5}\right)<1
$$

$\widetilde{\mathrm{v}}_{\mathrm{r}} / \overline{\mathrm{u}}_{\mathrm{s}}=0.055\left(\mathrm{x} / \Lambda \mathrm{We} \mathrm{e}_{\mathrm{f \Lambda}}^{0.5}\right)^{-0.78}, \mathrm{x} /\left(\Lambda \mathrm{We} \mathrm{e}_{\mathrm{f \Lambda}}^{0.5}\right)>1$

The value of $\widetilde{v}_{\mathrm{r}} / \overline{\mathrm{u}}_{\mathrm{s}}$ given in Eq. $9,0.055$, has a standard deviation of $21 \%$. The correlation coefficient of Eq. 9 is 0.90 and the standard deviation of the coefficient and power in Eq. 3.18 are $9 \%$ and $24 \%$, respectively. The value of $\widetilde{v}_{r} / \vec{u}_{s}$ near the jet exit, 0.055 , is comparable to 
cross stream rms velocity fluctuations not too near the wall for fully-developed turbulent pipe flow ${ }^{23}$ as might be expected for conditions where surface tension forces at the liquid surface are rather small compared to the momentum of eddy sizes responsible for the creation of drops by turbulent primary breakup.

\section{Drop liquid fluxes}

The last liquid surface property that was studied during the present investigation was the radial flux of drop liquid relative to the liquid surface as a result of turbulent primary breakup along the liquid surface, or $\dot{\mathrm{m}}_{\mathrm{f}}^{\prime \prime}$. In order to normalize this variable, it was defined in terms of the liquid surface breakup efficiency factor, $\varepsilon$, which is defined as follows:

$$
\varepsilon=\dot{\mathrm{m}}_{\mathrm{f}}^{\prime \prime} /\left(\rho_{\mathrm{f}} \widetilde{\mathrm{v}}_{\mathrm{r}}\right)
$$

where the limit $\varepsilon=1$ represents conditions where liquid drops are forming in a continuous manner over all the projected surface area of the liquid surface. The actual appearance of turbulent primary breakup, as the result of the Rayleigh-breakup of the tips of growing ligaments along the surface, however, suggests that generally $\varepsilon<1$, if not $\ll<1$.

Present measurements of $\varepsilon$ for turbulent primary breakup of turbulent annular and round liquid jets in still air are illustrated in Fig. 10. The independent variable of this figure is $\mathrm{x} /\left(\Lambda \mathrm{We}_{\mathrm{f \Lambda}}^{1 / 2}\right)$, which is the characteristic streamwise variable used to correlate the SMD after turbulent primary breakup as a function of streamwise distance. Limits giving the onset of turbulent primary breakup as well as the liquid column breakup length are also shown on the plot to help define the region of the liquid column where turbulent primary breakup was occurring. The correlation of the present measurements of the surface efficiency factor appearing in Fig. 10 yields a simple empirical power law relationship for $\varepsilon$ over the present test range, as follows:

$$
\varepsilon=0.016\left(\mathrm{x} /\left(\Lambda \mathrm{We}_{\mathrm{f \Lambda}}^{1 / 2}\right)\right)
$$

where actual best-fit power of the independent variable of Eq. 11 is 0.97 with experimental uncertainty $(95 \%$ confidence) of 0.03 which is not statistically different from unity; therefore, the simple form presented in Eq. 11 has been retained for the present data correlation. The standard deviation of the coefficient and power are $3 \%$ and $9 \%$, respectively and the correlation coefficient of fit equal to 0.92 , which is good. Moreover, the trend of the measurements illustrated in Fig. 10 appears to be quite reasonable.

\section{CONCLUSIONS}

The major conclusions of the present study concerning the surface breakup properties of fully-developed turbulent round and annular free liquid jets are as follows:

1. Drop formation from the tip of ligaments occurs by the classical Rayleigh breakup mechanism and is the dominant mechanism of drop formation (roughly $90 \%$ of the time). The remaining mechanism of drop formation (roughly $10 \%$ of the time) involves ligament breakup at its root due to cross stream velocity fluctuations near the liquid surface.

2. Drop velocity distributions after turbulent primary breakup satisfied uniform distribution functions; this is helpful because drop velocities are fully defined by single moments.

3. Mean and fluctuating drop velocities after turbulent primary breakup could be related quite simply to mean and fluctuating streamwise velocities in the liquid. Velocity properties were similar to recent observations of plane wall jets except for mean cross stream velocities, which decrease with increasing streamwise distance rather than remaining constant similar to wall jets.

4. The mean drop mass fluxes due to turbulent primary breakup at the liquid surface were correlated by defining a surface efficiency factor $(\varepsilon)$ in the same manner as the variation of drop SMD after turbulent primary breakup. Quite plausibly, $\varepsilon$ is small at the onset of turbulent primary breakup but reaches values on the order of magnitude of unity as the end of the liquid core is approached.

\section{ACKNOWLEDGMENTS}

This research was sponsored by the Office of Naval Research, Grant No. N0014-95-1-0234 under the technical management of E. P. Rood. Initial development of research facilities was carried out under Air Force Office of Scientific Research Grant No. AFOSR 49620-95-1-0364 under the technical management of J. M. Tishkoff.

\section{REFERENCES}

1. De Juhasz, K.J., Zahn, O.F., Jr., and Schweitzer, P.H., Pennsylvania State University, Engr. Exp. Station Bulletin No. 40 (1932).

2. Lee, D.W., and Spencer, R.C., NACA Tech. Notes 424 and 454, Washington (1933).

3. Schweitzer, P. H., J. App. Phys. 8, 513-521 (1937).

4. Grant, R. P. and Middleman, S., A.I.Ch.E. J. 12, 669678 (1966).

5. Phinney, R. E., J. Fluid Mech. 60, 689-701 (1973).

6. McCarthy, M. J. and Molloy, N. A., Chem. Eng. J. 7, 1-20 (1974). 
7. Hoyt, J. W., and Taylor, J. J., Phys. Fluids 20, S253S257 (1977).

8. Hoyt, J. W. and Taylor, J. J., J. Fluid Mech. 83, 119 127 (1977).

9. Ruff, G. A., Sagar, A. M. and Faeth, G. M., AIAA J. 27, 901-908 (1989).

10. Ruff, G. A., Bernal, L. P. and Faeth, G. M., J. Prop. Power 7, 901-908 (1991).

11. Ruff, G. A., Wu, P.-K., Bernal, L. P. and Faeth, G. M., J. Prop. Power 8, 280-289 (1992).

12. Tseng, L.-K., Ruff, G. A. and Faeth, G. M., AIAA J. 30, 1537-1544 (1992).

13. Wu, P.-K., Tseng, L.-K., and Faeth, G.M., Atom. Sprays 2:295-317 (1992).

14. Wu, P.-K., and Faeth, G.M., Atom. Sprays 3, $265-$ 289 (1993).

15. Wu, P.-K., Miranda, R.F., and Faeth, G.M., Atom. Sprays 5, 175-196 (1995).

16. Wu, P.-K., and Faeth, G.M., Phys. of Fluids A, 7, 2915-2917 (1995).

17. Dai, Z., Hsiang, L.-P. and Faeth, G. M., Proceedings of the $21^{\text {st }}$ Symposium on Naval Hydrodynamics, National Academy Press, Washington, D.C., 490-505 (1997).

18. Dai, Z., Chou, W.-H. and Faeth, G. M., Phys. Fluids 10, 1147-1157 (1998).

19. Simmons, H. C., J. Eng. Power 99, 309-319 (1977).

20. Merrill, C. F. and Sarpkaya, T., AIAA paper No. 980442 (1998).

21. Tyler, E., Phil. Mag. 16, 504-518 (1933).

22. Weber, C., Z. Angewesen. Math. Mech. 2, 136-141 (1931).

23. Tennekes, H., and Lumley, J.L., A First Course in Turbulence, MIT Press, Cambridge, MA, 248-296 (1972).

Table 2 Summary of test conditions for annular free jet ${ }^{\mathrm{a}}$

\begin{tabular}{|l|l|l|}
\hline Parameter & Formula & Range \\
\hline $\mathbf{b}(\mathrm{mm})$ & & $1,3.55,6.75$ \\
\hline $\mathrm{u}_{\mathrm{o}}(\mathrm{m} / \mathrm{s})$ & & $11.1-31.2$ \\
\hline $\mathrm{Re}_{\mathrm{fd}}$ & $\rho_{\mathrm{f}} \mathrm{u}_{\mathrm{o}} \mathrm{d}_{\mathrm{h}} / \mu_{\mathrm{f}}$ & $25,000-424,000$ \\
\hline $\mathrm{We}_{\mathrm{fd}}$ & $\rho_{\mathrm{f}} \mathrm{u}_{\mathrm{o}}{ }^{2} \mathrm{~d}_{\mathrm{h}} / \sigma$ & $3,000-151,000$ \\
\hline $\mathrm{Oh}_{\mathrm{fd}}$ & $\mu_{\mathrm{f}} /\left(\rho_{\mathrm{f}} \mathrm{d}_{\mathrm{h}} \sigma\right)^{1 / 2}$ & $0.00091-0.00336$ \\
\hline
\end{tabular}

${ }^{\mathrm{a} A n n u l a r}$ water jet in air at $100 \mathrm{kPa}$ and $297 \pm 0.5 \mathrm{~K}$. Properties of air as mentioned in Table 1. Hydraulic diameter taken as $2 b$.
Table 1 Summary of test conditions for round free jet ${ }^{\mathrm{a}}$

\begin{tabular}{|l|l|l|}
\hline Liquid & Water & Ethanol \\
\hline$\rho_{\mathrm{f}}\left(\mathrm{kg} / \mathrm{m}^{3}\right)$ & 997 & 800 \\
\hline$\rho_{\mathrm{f}} / \rho_{\mathrm{g}}$ & 860 & 690 \\
\hline$\mu_{\mathrm{f}} \times 10^{4}(\mathrm{~kg} / \mathrm{ms})$ & 8.94 & 16.0 \\
\hline$\sigma \times 10^{3}(\mathrm{~N} / \mathrm{m})$ & 70.8 & 24.0 \\
\hline $\mathrm{d}(\mathrm{mm})$ & $1.9,4.8,8.0$ & 4.8 \\
\hline $\mathrm{u}_{\mathrm{o}}(\mathrm{m} / \mathrm{s})$ & $3-25$ & $23-30$ \\
\hline $\mathrm{Re}_{\mathrm{fd}} \times 10^{-3}$ & $6-136$ & $57-129$ \\
\hline $\mathrm{We}_{\mathrm{fd}} \times 10^{-2}$ & $2-400$ & $500-3000$ \\
\hline $\mathrm{Oh}_{\mathrm{fd}} \times 10^{4}$ & $15-24$ & 53 \\
\hline $\mathrm{L}_{\mathrm{c}} / \mathrm{d}$ & $50-300$ & $200-300$ \\
\hline
\end{tabular}

${ }^{2}$ Pressure-atomized injection vertically downward in still air at $99 \pm 0.5 \mathrm{kPa}$ and $297 \pm 0.5 \mathrm{~K}\left(\rho_{\mathrm{g}}=1.16\right.$ $\mathrm{kg} / \mathrm{m}^{3}$ and $v_{\mathrm{g}}=15.9 \mathrm{~mm}^{2} / \mathrm{s}$ ). Round injector with a rounded entry and a length-to-diameter ratio of 40:1.

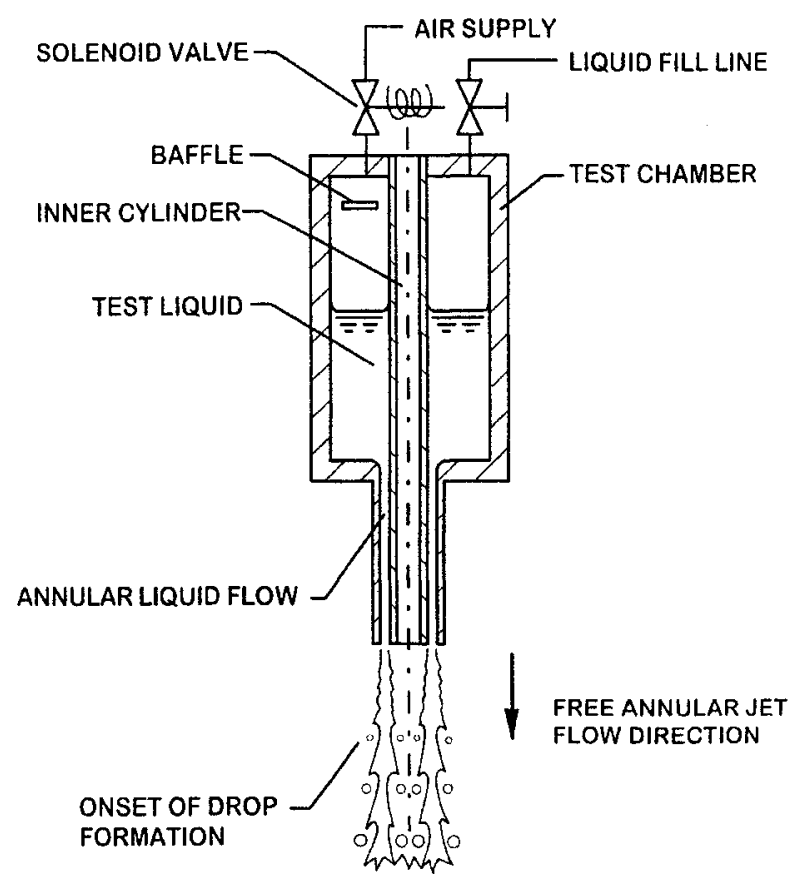

Fig.1 Sketch of the annular jet apparatus. 


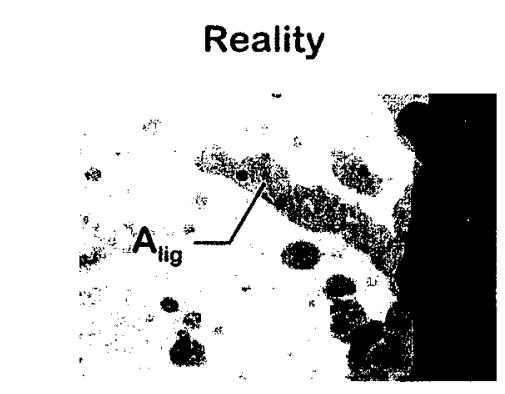

Idealization

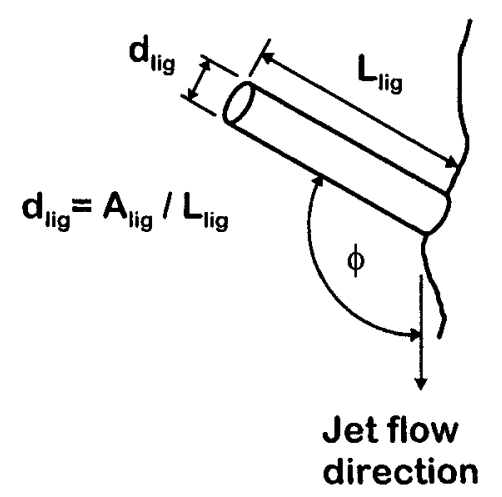

Fig.2 Sketch of the definitions of ligament length, $L_{\text {lig, }}$, ligament effective diameter, $\mathrm{d}_{\mathrm{lig}}$, and ligament angle, $\phi$.

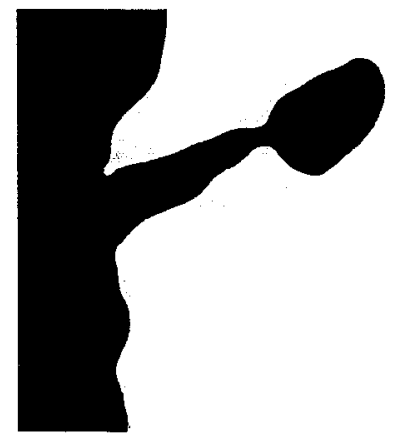

Fig.4 Typical shadowgraph of ligament tip (Rayleigh) breakup at the surface of round and annular free jets.

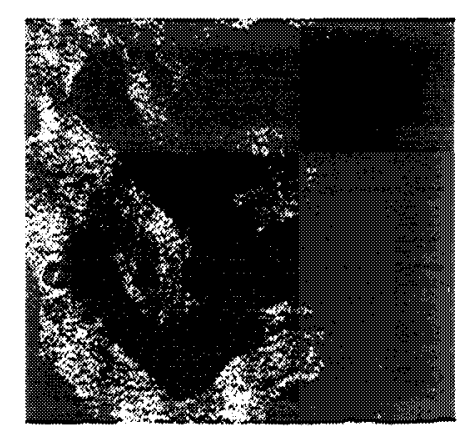

Fig.5 Typical double-pulse shadowgraphs of ligament base (turbulent) breakup at the surface of round and annular free jets.

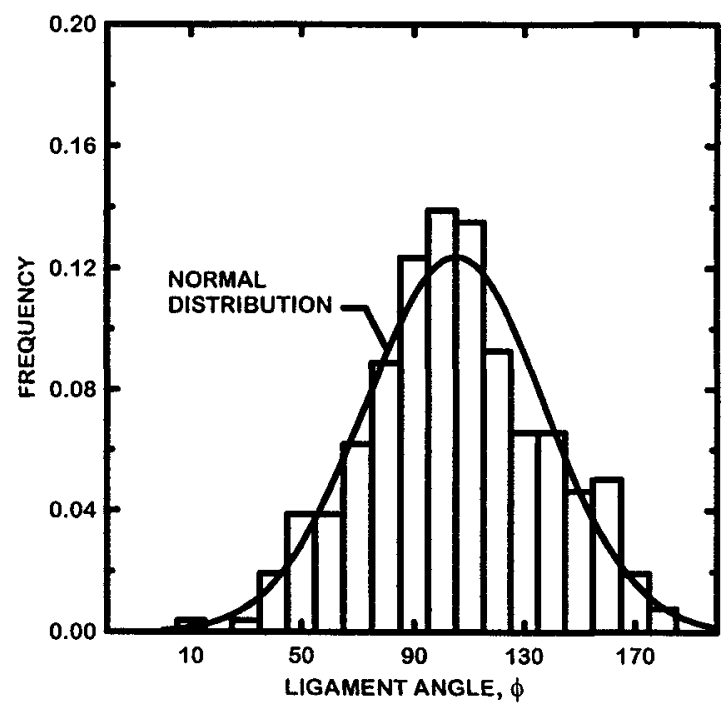

Fig.3 Ligament angle, $\phi$, probability density distribution for annular and round free jets.

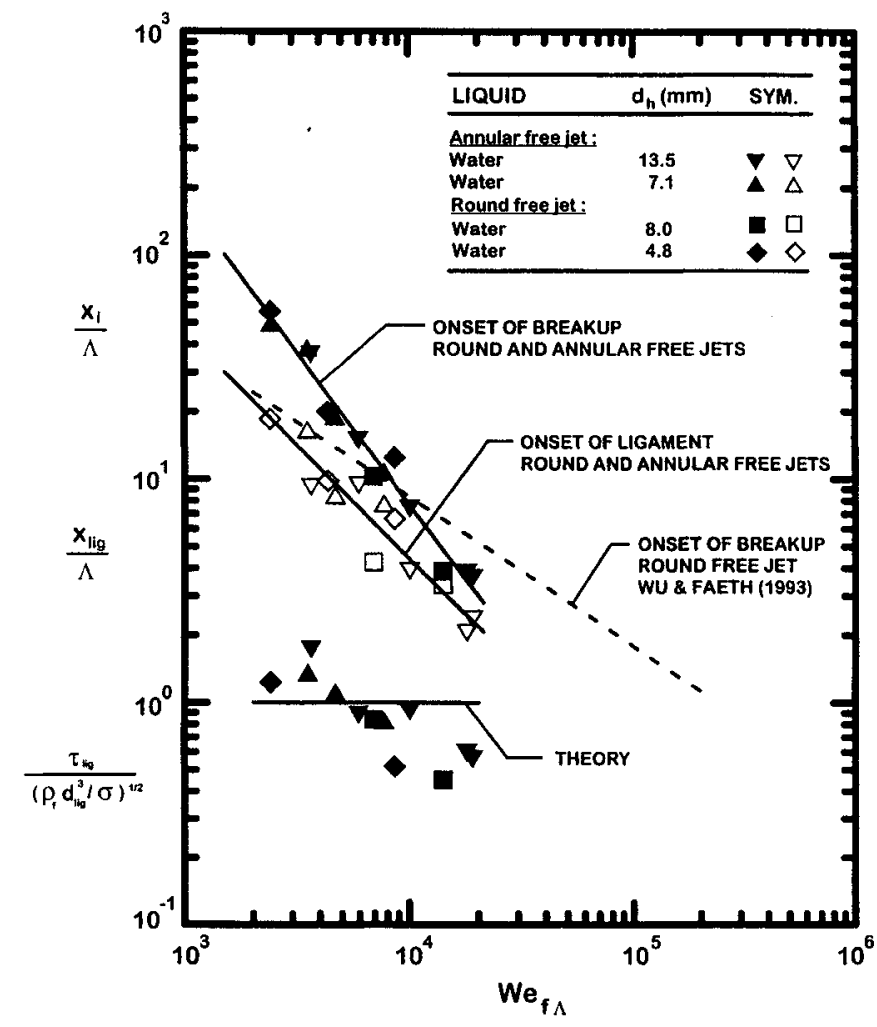

Fig.6 Length to initiate ligament and drop formation for round and annular free jets as a function of Weber number; and ligament breakup times as a function of Weber number for round and annular free jets. 


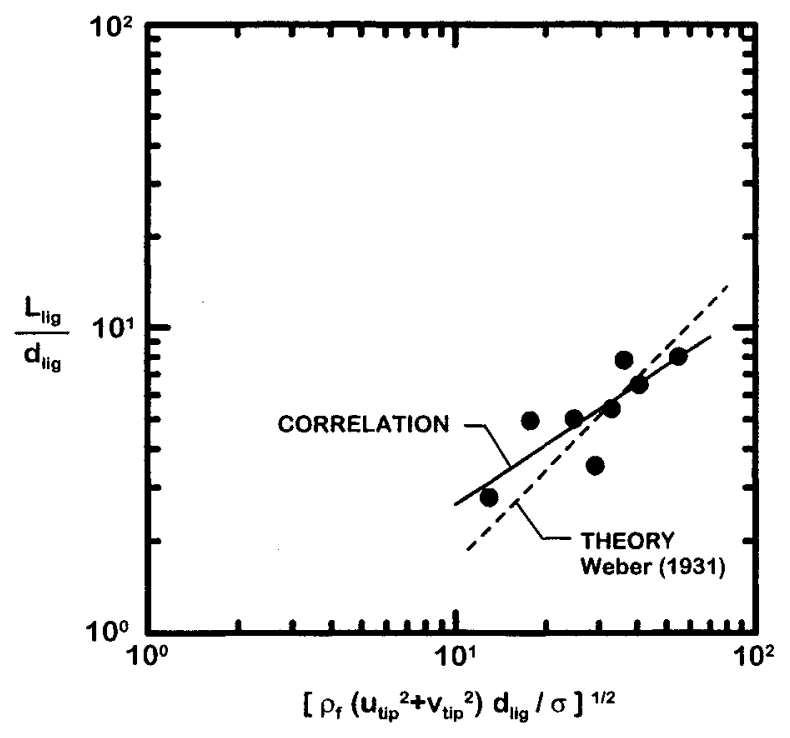

Fig. 7 Ligament slenderness ratios at breakup plotted as a function of the Weber number based on the ligament tip velocity and ligament diameter.

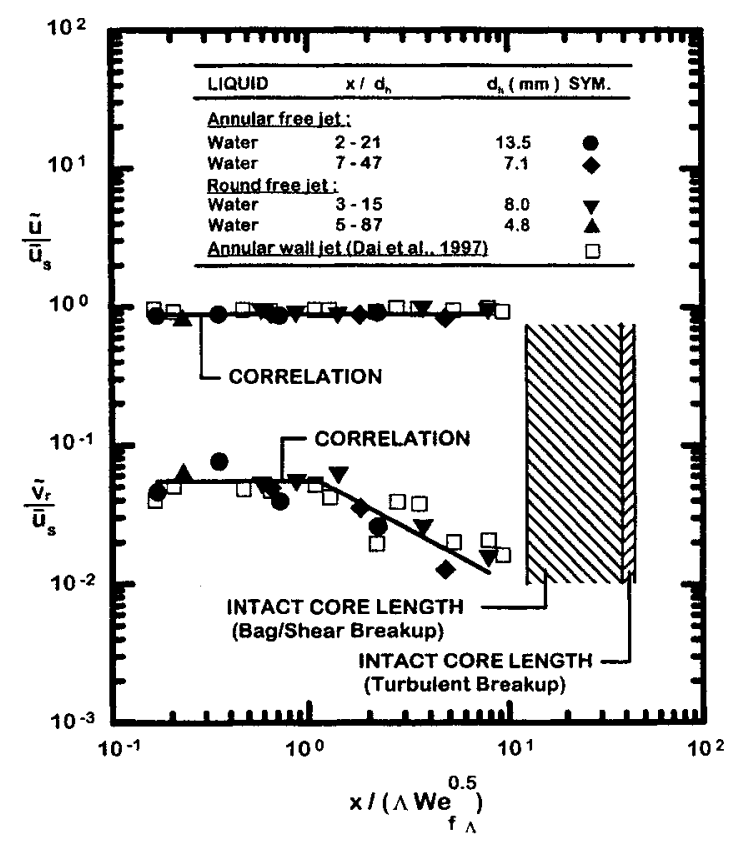

Fig.9 Absolute mean streamwise and relative cross stream drop velocities as a function of normalized streamwise distance from the jet exit.

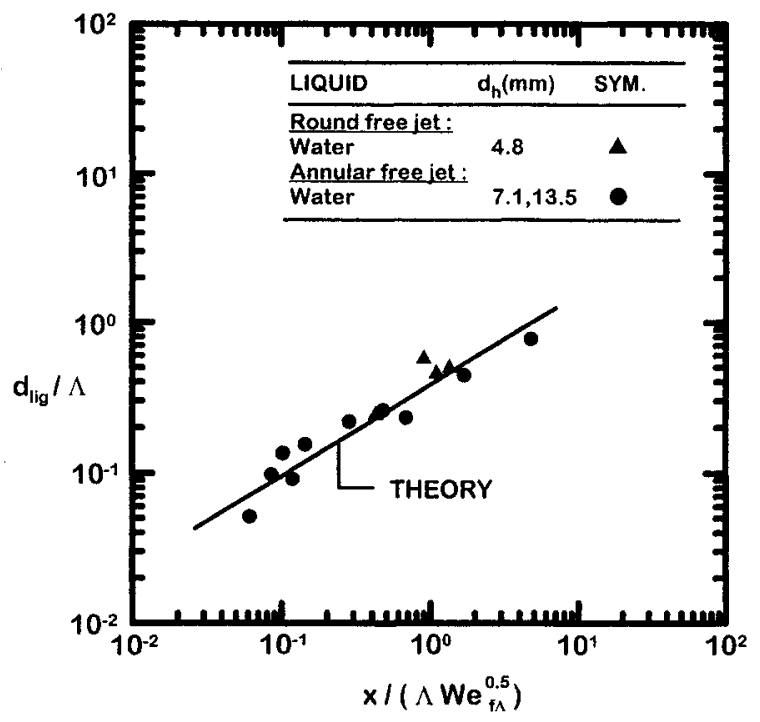

Fig.8 Ligament size as a function of the normalized downstream distance along the surface of round and annular free jets.

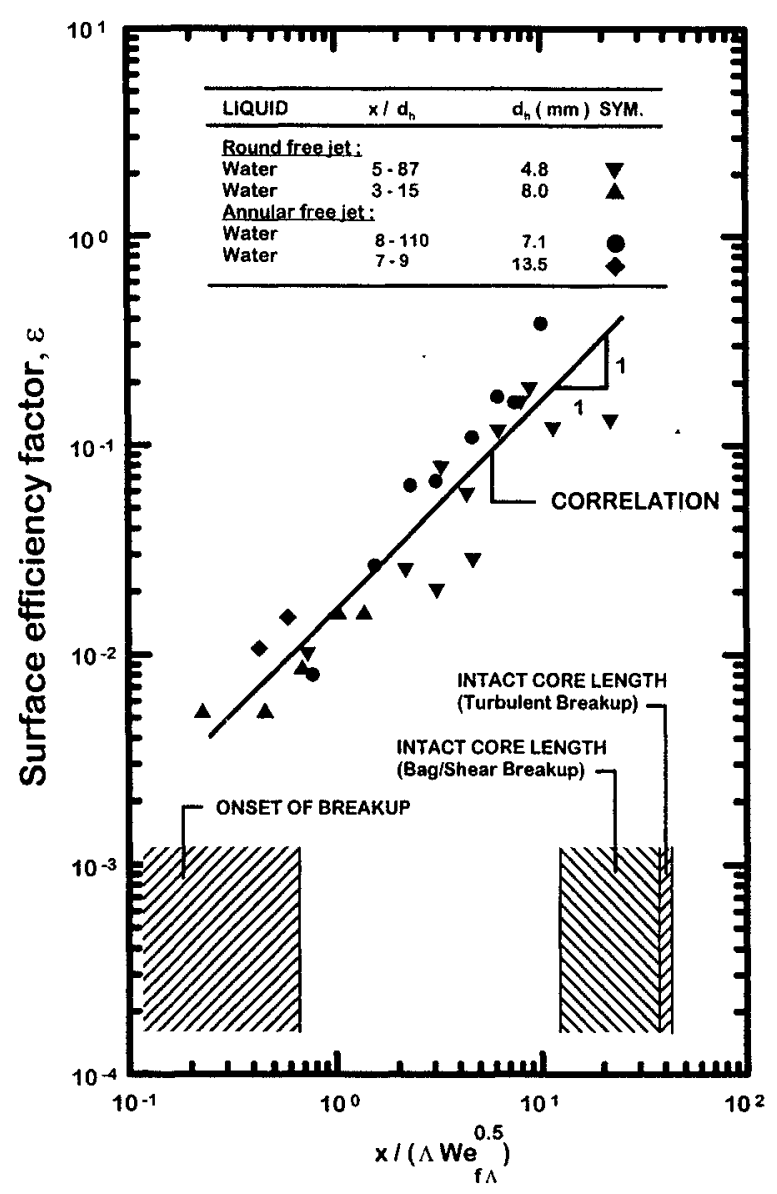

Fig.10 Mean surface efficiency factors as a function of normalized streamwise distance from the jet exit for round free jets and annular free jets. 